\title{
Within-pair similarity in migration route and female winter foraging effort predict pair breeding performance in a monogamous seabird
}

\author{
Annette L. Fayet ${ }^{1, *}$, Akiko Shoji ${ }^{1}$, Robin Freeman ${ }^{2}$, Chris M. Perrins ${ }^{1}$, Tim Guilford ${ }^{1}$ \\ ${ }^{1}$ Department of Zoology, University of Oxford, Oxford OX1 3PS, UK \\ ${ }^{2}$ Institute of Zoology, Zoological Society of London, Regent's Park, London NW1 4RY, UK
}

\begin{abstract}
In long-lived monogamous animals, pair bond strength and durability are usually associated with higher fitness. However, whether pairs maximise fitness during the non-breeding season by maintaining contact during the winter or, instead, prioritise individual condition is unclear. Using geolocators recording spatial (light) and behavioural (immersion) data, we tracked pairs of the long-term monogamous Atlantic puffin Fratercula arctica during the non-breeding season to determine whether and how migratory strategies were related to future pair breeding performance and whether within-pair similarity in migratory movements or individual behaviour best predicted future fitness. While pair members migrated separately, their routes were similar in the first part of the non-breeding season but diverged later on; nonetheless, pairs showed synchrony in their return to the breeding colony in spring. Pairs following more similar routes bred earlier and had a higher breeding success the following spring. However, female (but not male) winter foraging effort was also a strong predictor of subsequent fitness, being associated with future timing of breeding and reproductive success. Overall, females had higher daily energy expenditure than males, especially in the late winter when their route diverged from their partner's and they foraged more than males. Our study reveals that female winter foraging, probably linked to pre-breeding condition, may be more critical for fitness than maintaining the pair bond outside of the breeding season. However, even without contact between mates, pairs can benefit from following similar migration routes and synchronise their returns, but the mechanisms linking these processes remain unclear.
\end{abstract}

KEY WORDS: Atlantic puffin - Energy expenditure - Foraging · Geolocation · Migration · Pair bond $\cdot$ Seabird

\section{INTRODUCTION}

Many long-lived animals, birds in particular, form monogamous, long-term pair bonds (Emlen \& Oring 1977, Clutton-Brock 1989, Black 1996). Reproductive success often increases with pair duration (Dubois \& Cézilly 2002, Sánchez-Macouzet et al. 2014), and changing mates is costly (Choudhury 1995). In migratory birds, males often return to the breeding grounds before their mate to defend a nest site (Newton 2008), and pairs with similar return dates tend to

${ }^{*}$ Corresponding author: annette.fayet@gmail.com have higher breeding success and lower divorce rates (González-Solís et al. 1999, Naves et al. 2007). In migratory species where partners winter far from the breeding grounds, pairs may have more difficulty in reuniting after migration (Rowley 1983). Little is known about whether and how migratory pairs maintain the pair bond outside of the breeding season or whether proximity to the partner only matters during breeding and prioritising individual condition is more important to ensure higher future breeding success. Maintaining contact by coordinating move-

(C) The authors 2017. Open Access under Creative Commons by Attribution Licence. Use, distribution and reproduction are unrestricted. Authors and original publication must be credited. 
ments can allow pairs to ensure the continuity of their pair bond (Ens et al. 1996). Alternatively, wintering in similar areas, thereby experiencing and reacting to similar environmental conditions, may enable partners to synchronise their returns to the breeding grounds and avoid mate infidelity (Handel \& Gill 2000) or divorce (González-Solís et al. 1999) without maintaining contact outside of the breeding season. A good body condition at the start of the breeding season may also be important to maximise breeding success (Chastel et al. 1995, Wendeln \& Becker 1999) but may necessitate partners to forage in different niches or places if their requirements differ. There is strong evidence, particularly in seabirds, for sexspecific energy requirements and foraging strategies during breeding (Lewis et al. 2002, Alves et al. 2013, Conners et al. 2015, Widmann et al. 2015), and this may also be the case outside of the breeding season, although perhaps to a lesser extent (Phillips et al. 2011). Therefore, ensuring pair bond continuity while foraging for one's own individual requirements may not be compatible. Whether and how pairs of monogamous birds prioritise one over the other or combine both are unknown.

Recent miniaturisation of tracking technology has enabled researchers to follow pairs of monogamous birds throughout the non-breeding season, but to date only a few studies have done so. Pairs of waders were found to winter far apart but still show synchronised returns to the breeding grounds, the mechanisms behind which remain unclear (Gunnarsson et al. 2004). Two species of long-lived monogamous seabirds, Scopoli's shearwaters Calonectris diomedea and southern rockhopper penguins Eudyptes chrysocome, both seem to migrate separately to mostly similar places, and penguin mates forage in different niches (Müller et al. 2015, Thiebot et al. 2015). However, whether these behaviours, such as following similar migration routes or foraging in separate niches, affected future fitness is unknown.

Here, we use geolocation tracking data, combined with individual at-sea activity budgets to provide estimates of daily behaviour and energy expenditure, to examine the within-pair dynamics of migration both spatially and behaviourally in a long-lived monogamous dispersive migrant, the Atlantic puffin Fratercula arctica (Guilford et al. 2011, Fayet et al. 2016). More specifically, we investigate how certain measures of a pair's reproductive success are related to the non-breeding behaviour of each mate and of the pair as a whole by testing (1) whether pair members migrate together or separately and synchronise their arrival to, and departure from, the colony and if there is any fitness advantage for mates to follow similar routes (in terms of timing of breeding and reproductive success); (2) whether sexes differ in their non-breeding behaviour (which would indicate distinct foraging strategies or niches that may promote separation of partners during non-breeding); and (3) the extent to which each pair member's foraging effort during the non-breeding season predicts the pair's subsequent breeding performance.

\section{METHODS}

\section{Ethical statement}

All work was approved by the British Trust for Ornithology Unconventional Methods Technical Panel (permit C/5311), Skomer Island Advisory Committee, Natural Resources Wales and the University of Oxford Local Ethical Review Process. To minimise disturbance to the birds, handling time was reduced to a minimum ( $<10 \mathrm{~min})$. No obvious impact was observed on any of the pairs, and all continued behaving normally after handling. The breeding success and survival of puffins tracked with geolocators on our study site are similar to control birds (Fayet et al. 2016).

\section{Study site and geolocation data collection}

This study was conducted on Skomer Island, Wales, UK $\left(51^{\circ} 44^{\prime} \mathrm{N}, 5^{\circ} 19^{\prime} \mathrm{W}\right)$. On Skomer, puffins return to the colony early in April and start laying eggs soon after, and incubation lasts until mid-May to early June. Chicks fledge from late June to midJuly, and soon after, the adults leave the colony and start their migration. Migratory patterns are very diverse and can include successive trips to different wintering sites (Fayet et al. 2016). The timings and locations of moult are unknown but likely to vary widely within populations (Harris et al. 2014). Between 2009 and 2015, 16 pairs of chick-rearing puffins were equipped with a geolocator on a leg ring (Biotrack, $\leq 2 \mathrm{~g}, \sim 0.5 \%$ of body mass) for 1 to $3 \mathrm{yr}$, recording light level and immersion. All birds were recaptured during the following breeding seasons to download data, but 10 geolocators failed to record data, reducing the number of pairs for which we collected data simultaneously to 12 , from which we obtained 20 pairs of tracks (including repeated tracks from 7 pairs). 


\section{Spatial data analysis}

The resolution of geolocation data is inherently low ( $185 \mathrm{~km}$, Phillips et al. 2004); therefore, the absolute values calculated in this study (e.g. distance covered) must be interpreted carefully. However, the spatial variables estimated are only used for comparison between individuals, so it is their relative value which is considered, which will be less affected by any systematic over- or under-estimation than the absolute values. We also processed the data to minimise error, using the following methods (described in Fayet et al. 2016). Two-day median positions were calculated from the geolocation light data for all migration tracks, using a speed filter of $500 \mathrm{~km} \mathrm{~d}^{-1}$ and removing data close to the equinoxes. The total distance covered by each bird on migration was calculated using greatcircle distances (shortest distance between 2 points measured on the surface of a sphere); this variable was calculated to be included in analyses of energy expenditure. To test whether partners followed similar routes, we calculated the average nearest-neighbour distance (hereafter NND, a variable in kilometers decreasing with route similarity; see Guilford et al. 2011 for detailed methods) of each track to its partner's track (group = partners) and to all other tracks of the opposite sex collected in the same year (group = non-partners). The NND was then compared between the 2 groups. The NND was calculated over a $20 \mathrm{~d}$ window to add a temporal aspect to our calculation of route similarity; in other words, the tracks of 2 birds visiting similar locations months apart would not count as being similar. As in Fayet et al. (2016), a $20 \mathrm{~d}$ window was chosen as a good compromise between short enough to compare synchrony between birds and long enough to ensure a reasonable range of locations from which to find a nearest neighbour (due to the spatial resolution of geolocation, there may be gaps in the data on particular days).

\section{Pair synchrony in departure and return dates}

Although the resolution of geolocation precludes determining whether partners with similar tracks actually migrated together, it is possible to identify the precise timing of large longitudinal movements, with within-pair differences in such timings indicating that birds are migrating separately. Nine of 12 pairs showed large westward movements to the middle or western North Atlantic shortly after the breeding season; for each member of these pairs, we identified the timing of crossing of the $-20^{\circ}$ meridian (an arbi- trarily chosen longitude which was crossed by all 9 pairs and sufficiently far from the colony to indicate a migratory movement). Timings of the first and last visits to the burrow were measured by using a combination of light and immersion data, identifying burrow visits as bouts of $10 \mathrm{~min}$ (the resolution of the immersion data) or more during daylight hours which were $>75 \%$ dry and $<100 \%$ light and were preceded by $5 \mathrm{~min}$ of light and dry (i.e. a $10 \mathrm{~min}$ bout with full daylight and $\geq 50 \%$ dry) corresponding to the birds standing on the colony prior to entering the burrow). This automated method only detected visits during the spring and summer months, confirming that such behaviour is indeed likely to be a burrow visit. The first of these, usually occurring in late March or early April, was considered the date of first return to the colony, while the last one, usually in July, was considered the date of departure from the colony. To test for synchrony between partners, we calculated and compared differences in timing of the first and last burrow visits between partners and between nonpaired birds of the opposite sex tracked during the same year.

\section{Behavioural data analysis}

Immersion data (the proportion of time spent immersed in salt water for each 10 min interval) were used to calculate the birds' daily activity budgets (duration and proportion of daylight hours spent flying, foraging and sitting on the water) and estimates of daily energy expenditure (DEE) as in Fayet et al. (2016). Briefly, each immersion data point collected during daylight between August and March was allocated to 1 of 3 categories: sustained flight ( $\geq 98 \%$ dry), sitting on the water surface ( $\geq 98 \%$ immersed) or foraging-related activity (hereafter foraging, $>2 \%$ dry and $>2 \%$ wet). The latter represents an alternation of short flight bouts (searching for prey) with short wet bouts (sitting or diving) indicative of foraging and was found to be strongly associated with intermediate speed and high tortuosity (area-restricted search) and diving behaviour in Manx shearwaters Puffinus puffinus (Dean et al. 2012), seabirds of similar size and relatively similar diving behaviour to puffins (Shoji et al. 2015, 2016). Energy budgets were estimated by combining these daily daytime budgets with nighttime budgets (resting or sleeping; see Section 1 of the Supplement at www.int-res.com/articles/ suppl/m569p243_supp.pdf for more details) and using a model of DEE developed for common guillemots Uria aalge (Elliott et al. 2013). The numbers were 
then scaled down for puffins with an allometric equation for auks (Shaffer 2011), using each bird's individual weight (obtained during chick rearing) for a more precise estimation (Supplement).

\section{Sex and breeding data collection}

Seven pairs captured in 2014 or later were sexed using DNA from feather samples. For licensing reasons, samples were not available for the 5 remaining pairs caught in previous years; 4 of these were sexed using a combination of behavioural and morphometrics measures (a method validated by DNA techniques in Fayet et al. 2016). One pair could not be sexed with confidence and was excluded from sexdifferences analyses. Pair fitness was measured in terms of timing of breeding (laying date) and breeding success. We obtained laying dates for each pair by combining the light data collected by geolocators during the incubation period (devices were only retrieved during chick rearing) to obtain the pair minimum light level every $5 \mathrm{~min}$. Since puffins take turns to incubate the egg continuously in the burrow for $\sim 40 \mathrm{~d}$, the incubation period was easily identifiable as a long dark period, whose first day would coincide with the start of incubation. This method was validated with burrow observations (Fayet et al. 2016). Breeding success (before and after migration) was obtained using observations of parents bringing fish to the burrow and observations of the nest with a burrowscope. Puffins provision their chick for $\sim 6 \mathrm{wk}$, but chicks are most vulnerable during their first few days, and mortality decreases sharply after that. Any pair managing to rear a chick for at least 2 wk (the majority of pairs were observed rearing their chicks for much longer) was considered successful.

\section{Statistical analyses}

Linear mixed-effects models (LMMs) were used throughout the analysis (using the lme4 package in $\mathrm{R})$, including year and either bird identity (when testing sex-specific variables, e.g. female foraging effort), pair identity (when testing pair-specific variables, e.g. lay date) or bird identity nested within pair identity (when both pair members were included) as random effects. Month was included as an additional explanatory variable when looking at temporal patterns of pair route similarity. Earlier findings suggest that individual puffins following different migratory routes have different breeding success, with birds wintering in the Mediterranean Sea doing best, followed by birds remaining fairly locally and then by birds only visiting the middle or western North Atlantic (Fayet et al. 2016). Therefore, we controlled for location (broad geographical area, i.e. North Atlantic, Mediterranean and local) and total distance covered when testing the relationship of within-pair route similarity on subsequent laying date. LMMs including sex and month as explanatory variables were used to study sex differences in DEE and behaviour throughout the winter. Location did not explain any variance in these models and was therefore not included.

\section{RESULTS}

In all years, birds followed a diversity of routes around the British Isles, to the middle or western North Atlantic and to the Mediterranean Sea (Fig. 1, Fig. S1 in the Supplement). As previously found at this colony (Guilford et al. 2011, Fayet et al. 2016), individual route fidelity between years was high and was higher than route similarity between partners and route similarity between non-partners (see Section 2 of the Supplement). Overwinter route similarity was higher between partners than non-partners, but the difference was not significant (partners: $\mathrm{NND}=667 \pm 85 \mathrm{~km}, \mathrm{n}=20$ pairs of tracks; nonpartners: NND $=708 \pm 37 \mathrm{~km}, \mathrm{n}=80$ pairs of tracks; LMM, $\chi^{2}{ }_{1}=0.15, \mathrm{p}=0.702$ ). However, we found a significant interaction between group (partner/nonpartner) and month when looking at the temporal patterns of NND throughout the non-breeding season $\left(\mathrm{LMM}, \chi^{2}{ }_{7}=20.4, \mathrm{p}=0.005\right)$, indicating that the difference between pair and non-pair route similarity varied depending on the time of the winter (Fig. 1d, Table S1 in the Supplement). Within-pair route similarity was high during the first 3 mo after breeding but gradually decreased until becoming no higher than non-partner route similarity in October and/or the rest of the winter. While in some pairs this decrease in within-pair route similarity was not accompanied by an obvious and sudden split in routes (Fig. S1), in one-third of pairs (4 of 12), a clear split occurred when 1 member flew to the Mediterranean Sea while the other remained in the Atlantic or near the French or Iberian coast (Fig. 1c). There was a slight increase in within-pair route similarity in February, prior to returning to the colony.

To determine whether pairs were likely to migrate together during the first part of the non-breeding season when within-pair route similarity was high, we used the 9 pairs (including repeat tracks for 4 

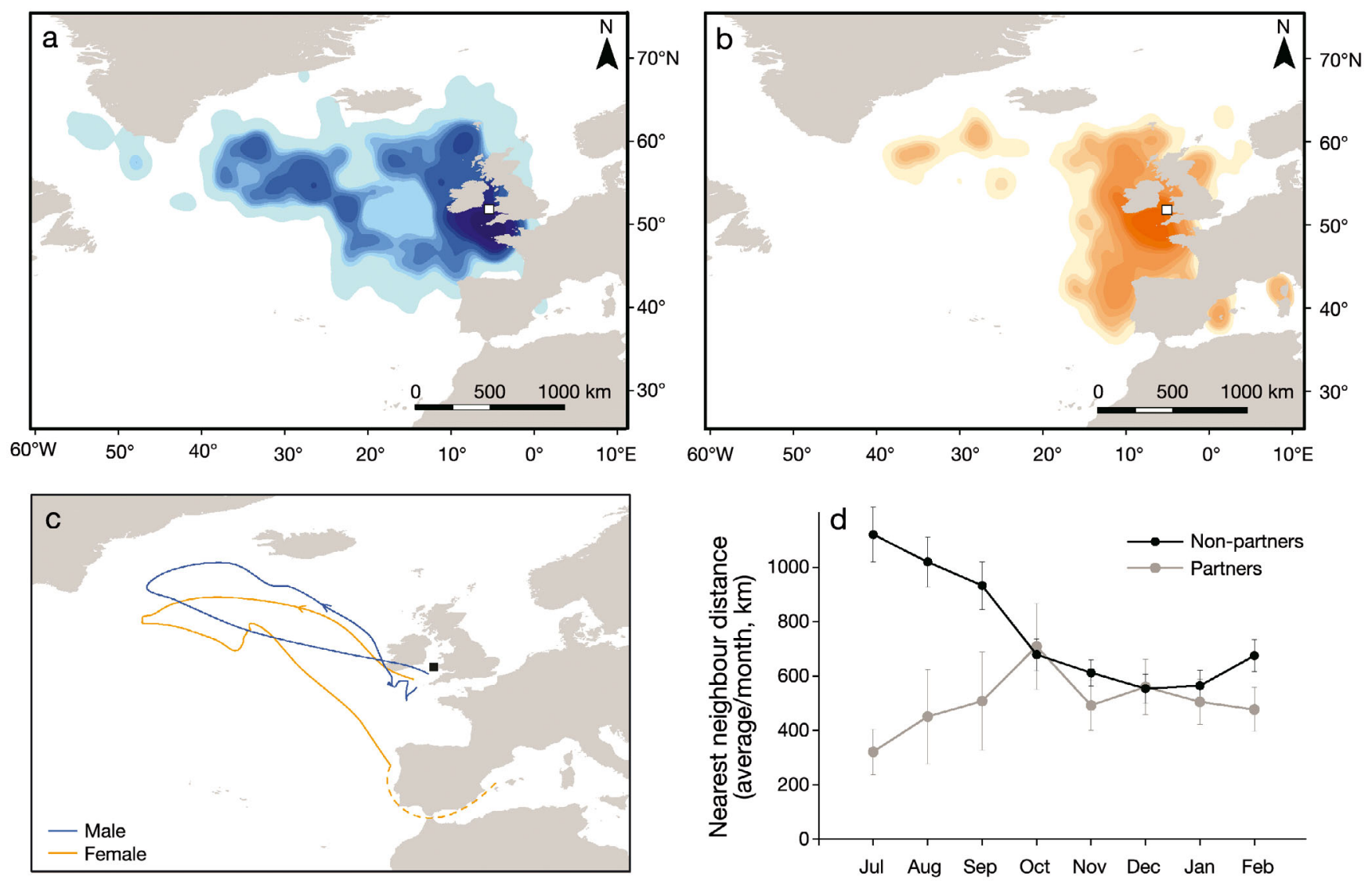

Fig. 1. Winter distribution of 12 pairs of puffins from Skomer Island, Wales, with (a) males in blue and (b) females in orange and $90 \%$ kernel density, with darker shades representing core areas (obtained using a bandwidth of $\sim 275 \mathrm{~km}$ and a resolution of $\sim 20 \mathrm{~km}$ ). The colony is indicated with a white square. (c) Example of a pair's migration (male in blue, female in orange, colony = black square). (d) Comparison of route similarity between partners (grey) and non-partners (black) by month $($ mean $\pm \mathrm{SE})$

pairs, i.e. 14 pair migrations in total) which showed large longitudinal westward movements to the Atlantic in July and August. In 9 of 14 cases, only 1 pair member crossed the $-20^{\circ}$ meridian. In the other 5 cases, pair members crossed the $-20^{\circ}$ longitude $7.6 \mathrm{~d}$ apart on average (range 2 to $18 \mathrm{~d}$ ). Taken together, these results indicate that most pairs migrate separately, despite following similar routes. Due to the poor latitudinal resolution of geolocation data, it is not possible to make conclusions on the 3 pairs which did not show large longitudinal movements. In spring, the timing of the first visit to the burrow was significantly more similar between partners than between non-paired birds of the opposite sex tracked during the same year (partners: $4.6 \pm 1.2 \mathrm{~d}$ apart, non-partners: $8.1 \pm 0.8 \mathrm{~d}$ apart; $\mathrm{LMM}, \chi^{2}{ }_{1}=4.5, \mathrm{p}=$ 0.035 ); in other words, partners returned to the colony more synchronously than non-partners. Unfortunately, due to the small number of pairs for which we had NND, lay date and timing of the first visit to the burrow ( 8 pairs over 4 yr), we could not test for potential relationships between NND and synchrony of return to the colony or between synchrony of return to the colony and subsequent lay date. At the end of the breeding season, the timing of the last visit to the burrow was not more similar between partners than between non-paired birds of the opposite sex tracked during the same year (partners: $3.2 \pm 0.7 \mathrm{~d}$ apart, non-partners: $3.7 \pm 0.4 \mathrm{~d}$ apart; $\mathrm{LMM}, \chi^{2}{ }_{1}=0.04, \mathrm{p}=0.839$ ); therefore, there was no particular pair synchrony in departure from the colony.

To test whether route similarity between mates was related to the pair's future fitness, we investigated the relationship between within-pair route similarity and subsequent laying date or breeding success. Pairs with more similar routes laid earlier the subsequent breeding season $(\mathrm{n}=19$, parameter estimate $=$ $9.18 \pm 5.47, \chi^{2}{ }_{1}=7.4, p=0.007$, controlling for location and total distance covered) and had a higher 
breeding success $\left(\mathrm{NND}_{\text {success }}=549 \pm 85 \mathrm{~km}, \mathrm{NND}_{\text {failure }}\right.$ $=726 \pm 389 \mathrm{~km}, \chi^{2}{ }_{1}=4.3, \mathrm{p}=0.038$, controlling for location and total distance covered). Prior breeding success did not predict within-pair route similarity the following winter $\left(\chi^{2}{ }_{1}=2.2, p=0.141\right.$, controlling for location and total distance covered).

Sexes differed in non-breeding behaviour and DEE. Females had a higher winter DEE after correcting for mass (females $=2.90 \pm 0.02 \mathrm{~kJ} \mathrm{~d}^{-1} \mathrm{~g}^{-1}$, males = $\left.2.78 \pm 0.02 \mathrm{~kJ} \mathrm{~d}^{-1} \mathrm{~g}^{-1}, \chi^{2}{ }_{1}=13.26, \mathrm{p}<0.001\right)$, despite both sexes covering similar total distances $\left(\chi^{2}{ }_{1}=0.77\right.$, $\mathrm{p}=0.379$ ). This was due to differences in overall winter activity budgets, with females spending similar amounts of time sitting on the water and foraging as males (sitting: $\chi^{2}=0.04, \mathrm{p}=0.837$; foraging, $\chi^{2}{ }_{1}=$ $0.10, \mathrm{p}=0.752)$ but more time flying $\left(\chi^{2}{ }_{1}=5.36, \mathrm{p}=\right.$ $0.021)$. To investigate these sex differences in energy expenditure in more detail, we compared the DEE of males and females throughout the winter by including month as an additional explanatory variable in the model (Fig. 2). DEE and all 3 types of behaviour varied significantly with month (DEE g ${ }^{-1}: \chi^{2}{ }_{6}=248.8$, $\mathrm{p}<0.001$; flying: $\chi^{2}{ }_{6}=40.8, \mathrm{p}<0.001$; foraging: $\chi^{2}{ }_{6}=$ 116.5, $\mathrm{p}<0.001$; sitting: $\left.\chi^{2}{ }_{6}=120.3, \mathrm{p}<0.001\right)$. For both sexes, DEE, flight and foraging were at their highest levels at the start of the non-breeding season, while sitting was at its lowest. Females had higher DEE (corrected for individual mass) than males throughout the winter (Fig. 2a). When looking at individual behaviours separately (Fig. $2 \mathrm{~b}-\mathrm{d}$ ), the proportions of time spent in each behavioural state were mostly similar between sexes. The main differences were that females tended to fly more than males from November onwards and to spend more time foraging and less time sitting in the later part of the winter (especially in January).

To test the extent to which non-breeding behaviour predicted future breeding performance, we investigated the relationship between foraging effort (proportion of daytime spent foraging) - at the individual and pair level — and subsequent lay date or breeding success. We found no correlation between previous breeding success and subsequent winter foraging effort in either sex (males: $544.7 \pm 65.2 \mathrm{~h}$ [success] vs. $482.6 \pm 49.7 \mathrm{~h}$ [failure]; females: $545.8 \pm 65.8 \mathrm{~h}$ [success] vs. $561.1 \pm 163.5 \mathrm{~h}$ [failure], $\chi^{2}{ }_{1}=0.12, \mathrm{p}=$ 0.943). Total pair foraging effort (the sum of each pair member's effort) seemed to predict subsequent breeding success, with pairs foraging more being more successful $\left(\chi_{1}^{2}=26.4, p<0.001\right)$. This was mainly due to an effect in females, with successful females foraging more than unsuccessful ones the previous winter $\left(601.2 \pm 70.9\right.$ vs. $203.5 \pm 9.9, \chi^{2}{ }_{1}=$
28.6, $\mathrm{p}<0.001$ ), while there was no significant difference between males $(534.5 \pm 60.3$ vs. $572.9 \pm 49.9$, $\chi_{1}^{2}=0.003, p=0.950$ ). Female foraging effort was also negatively correlated with lay date the following season (Fig. 2, parameter estimate $=-17.8 \pm 7.8, \chi^{2}{ }_{1}=$ 5.0, $p=0.025$ ): females which foraged more in winter laid earlier the following spring. This effect was not visible in males (parameter estimate $=4.1 \pm 9.9, \chi^{2}{ }_{1}=$ $0.19, \mathrm{p}=0.660$ ). There was no significant relationship between partners' foraging efforts (parameter estimate $=-0.27 \pm 0.27, \chi^{2}{ }_{1}=0.06, p=0.809$ ). Route similarity did not predict female foraging behaviour (parameter estimate $=-0.12 \pm 0.10, \chi^{2}{ }_{1}=1.7, \mathrm{p}=$ 0.196).

\section{DISCUSSION}

In this study, we investigated spatial and behavioural within-pair similarities in migratory strategies in puffins and examined the relationship between the pair and the individual non-breeding behaviour of each sex and future breeding performance. Regardless of previous breeding success, within-pair route similarity was high during the first part of the non-breeding season and gradually decreased until becoming similar to that of non-partners (from October onwards). However, despite this high within-pair route similarity in the first part of migration, pair members did not show synchrony in departure date from the colony and migrated separately, ruling out that within-pair route similarity is simply driven by similarity in departure date from the colony; indeed, other non-paired birds left at similar times (on average, all birds left within $3.6 \mathrm{~d}$ of each other across the whole dataset) and followed very different routes. This supports the results of 2 previous studies tracking seabird mates outside of the breeding season, which found that pairs of Scopoli's shearwaters and southern rockhopper penguins did not migrate together, despite following similar routes in some cases (Müller et al. 2015, Thiebot et al. 2015). While it was not investigated in these studies, here we tested whether within-pair route similarity was related to subsequent breeding timing and performance. In puffins, as in many other seabirds, early breeding is strongly associated with reproductive success (Nettleship 1972, Harris 1980), but lay date is not genetically determined (Harris \& Wanless 2011). We found that puffin pairs which followed more similar routes overall during the non-breeding season, regardless of wintering location or total distance covered during non-breeding, started to breed earlier 
a) DEE

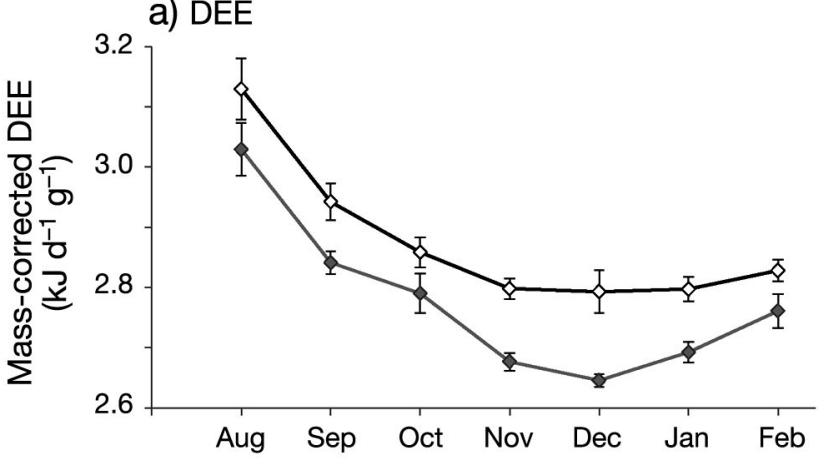

c) sitting on the water
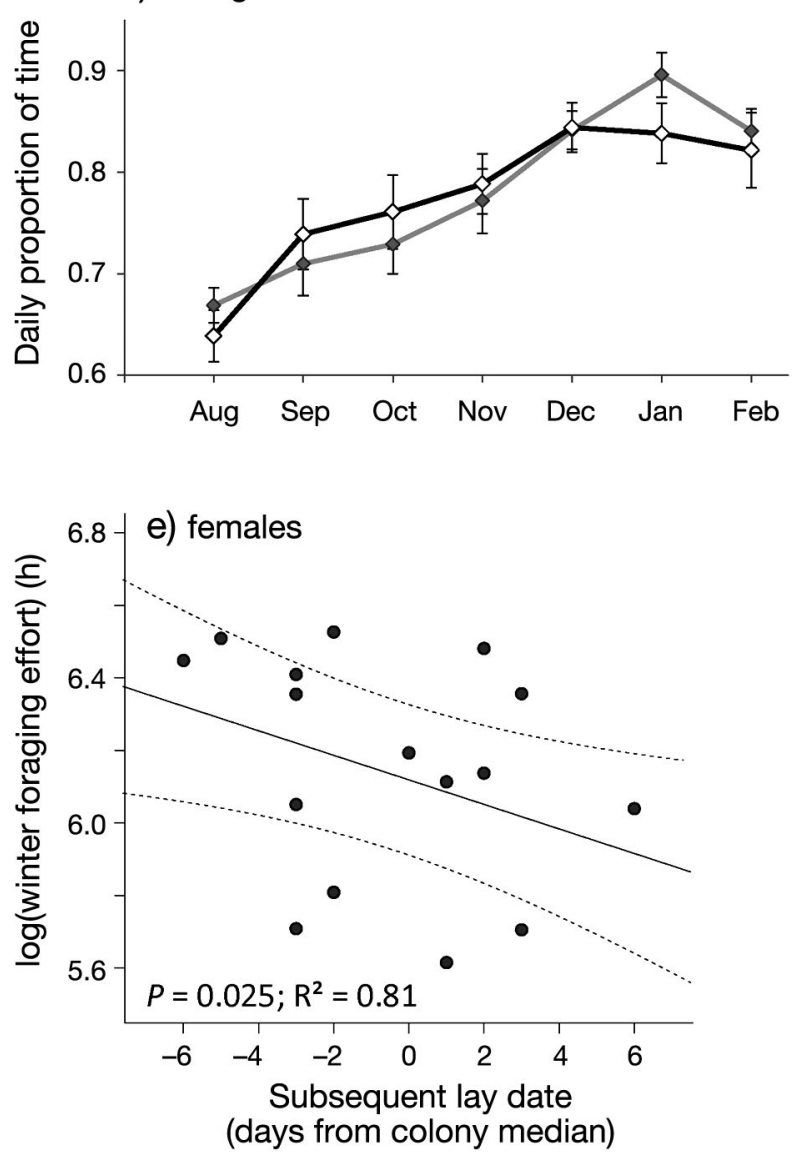

b) flying

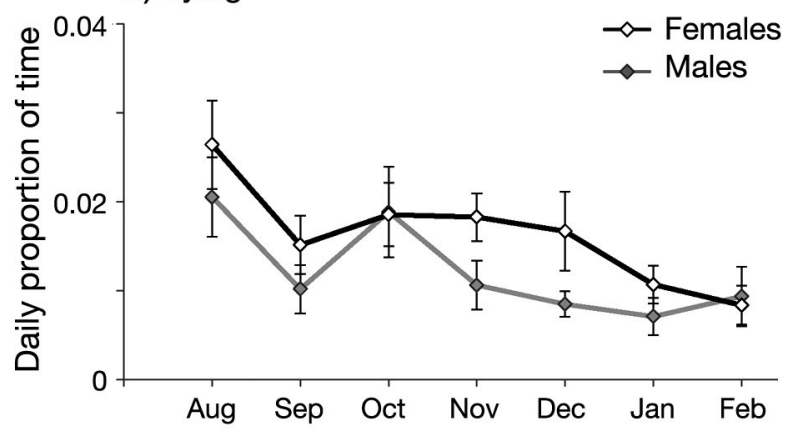

d) foraging
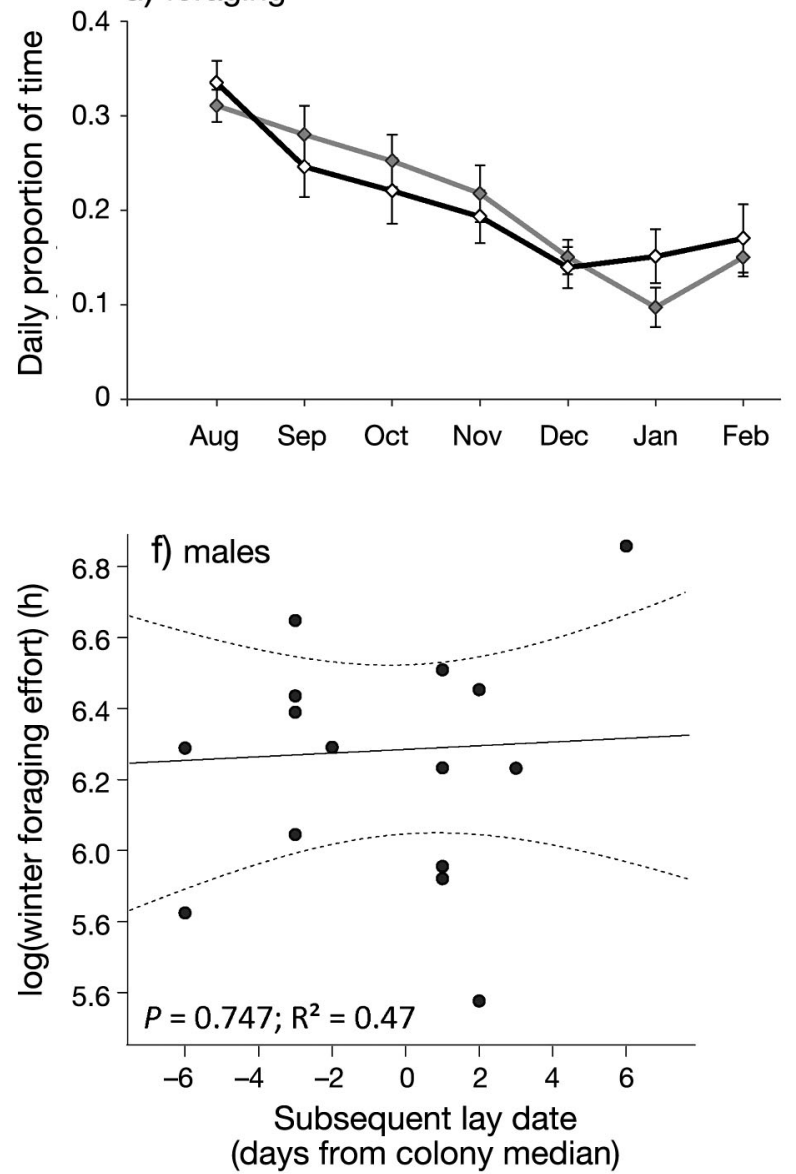

Fig. 2. Monthly averages (mean $\pm \mathrm{SE}$ ) of (a) daily energy expenditure (DEE) (corrected for individual mass) and proportion of time spent (b) flying, (c) sitting on the water and (d) foraging for males (grey) and females (black and white). Relationship between (e) female and (f) male winter foraging effort and subsequent lay date, with the regression lines obtained from linear mixed-effects models (see main text for statistics)

the following spring and had a higher breeding success. This suggests that following similar routes during non-breeding may increase a pair's fitness. Why this is the case remains unclear, but following similar migration routes could perhaps help earlier breeding by allowing pairs to synchronise their return to the colony. Indeed, we found that pair members were more synchronous in the timing of their first visit to the burrow (which we assume is a proxy for the timing of return to the colony) than non-paired birds. Pair synchrony in arrival at the breeding site is important for maintaining the pair bond in many 
migratory species including seabirds (González-Solís et al. 1999, Handel \& Gill 2000). Even without maintaining contact throughout the non-breeding season, exposure to similar environmental conditions at the wintering grounds may lead to similarity in timing of return to the breeding grounds, as suggested by the correlation between timing of individual arrival at the breeding grounds and winter habitat quality found in black-tailed godwits Limosa limosa (Gunnarsson et al. 2006). However, the spatial differences between partners in the second part of the winter make this hypothesis unlikely in puffins. Indeed, pairs may have 1 member in the Bay of Biscay and the other in the Mediterranean Sea in late winter, and these will likely experience different conditions, as suggested by the finding that puffins in the Mediterranean Sea spend more time foraging than puffins remaining in the North Atlantic in late winter (Fayet et al. 2016) (location did not explain variation in behaviour in this study, perhaps because of the smaller sample size). Nevertheless, synchronised returns to the breeding area can also occur in species where pairs migrate to different destinations, although the mechanisms remain unclear (Gunnarsson et al. 2004). Unfortunately, we did not have enough data to test directly the effect of pair route similarity on subsequent synchrony in return to the colony or the effect of pair synchrony of return to the colony on subsequent breeding performance. The emergence of spatial differences between partners in the second part of the winter (from October onwards) was shortly followed by increasing sex differences in DEE (female DEE was consistently higher throughout the winter, but this was more marked between November and January) and the emergence of sex differences in foraging and sitting behaviour, with females spending more time foraging and less time sitting than males in the late winter, in particular in January. Females also spent more time in flight over winter, despite covering similar total distances to males, and this seemed particularly marked in mid-winter (November to December), just after pair members started to follow less similar routes. While this apparent mismatch between flight time and distance may be due to inaccuracy in estimating distance from geolocation data, it could also result from sex differences in flight efficiency at certain wind speeds (Lewis et al. 2015, Cornioley et al. 2016) or exposure to different wind conditions when sexes visit different areas.

One of our key results is that while pair total foraging effort was a good predictor of future breeding performance (higher foraging effort was associated with earlier breeding), this was mainly due to the effect in females. Indeed, pairs whose female foraged more over winter started breeding earlier and had a higher breeding success the following summer, suggesting that female winter foraging effort is critical for subsequent reproductive performance. Taken separately, male winter foraging effort was not related to a pair's subsequent start of breeding or breeding success. Female foraging effort was not predicted by within-pair route similarity, suggesting that our results that both variables predict future lay date and breeding success may not be linked, but larger sample sizes may be needed to validate this result. While the mechanisms leading to these sex differences remain unknown, these results suggest that female winter foraging may be proactive, with females preparing for the next breeding season by foraging more, and that the adult condition at the start of breeding (presumably related to the amount of winter foraging) is more critical in females than in males for subsequent breeding success, perhaps because of egg laying. Although there was no clear spatial segregation between sexes at any point during the winter, puffin females may prepare for egg laying by splitting from their partner's route in late winter to visit more productive areas or forage on different prey types to satisfy different nutritional requirements, a hypothesis supported by the higher female DEE and foraging effort compared to males during that period. Sex differences in migratory niche exist in migratory birds including seabirds, not necessarily accompanied by spatial segregation (Alves et al. 2013, Thiebot et al. 2015), and chemical analyses of Brünnich's guillemots' eggs suggested that females relied on non-local energy sources to form their first (but not replacement) egg (Jacobs et al. 2009). In large auks like guillemots and puffins, the pre-laying exodus is either non-existent or too short to build an egg (Birkhead \& Nevo 1987), so accumulating reserves before returning to the colony may be an alternative way for females to prepare for egg laying.

Taken together, our results suggest that long-lived monogamous migratory birds benefit from prioritising individual condition over the pair bond during the non-breeding season, with female winter foraging effort, probably related to pre-breeding condition, being particularly important to future breeding performance. Maintaining contact outside of the breeding season does not seem necessary to ensure subsequent reproductive success. However, although the mechanisms remain unclear, following similar migration routes to one another seems to allow pairs to breed earlier and more successfully the following 
spring, and pair synchrony in return to the breeding grounds may occur even if partners winter in different areas. These results have important implications for sexual conflict, mate retention and sex differences in migration costs.

Acknowledgements. We thank D. Boyle, J. Roberts, P. Collins, H. Kirk, O. Padget, B. Dean and many others for their help in the field; the Skomer wardens C. Taylor, B. Büche and E. Stubbings and Natural Resources Wales for allowing us to carry out this study on Skomer Island; and OxNav colleagues and 3 anonymous reviewers for their useful comments on earlier versions of this manuscript. A.L.F. was funded by scholarships from the Biotechnology and Biological Sciences Research Council, Microsoft Research Cambridge, the Mary Griffiths Foundation and an award from the British Federation for Women Graduates. This work was funded by Microsoft Research Cambridge, the Department of Zoology of Oxford University and the Royal Society for the Protection of Birds.

\section{LITERATURE CITED}

Alves JA, Gunnarsson TG, Potts PM, Sutherland WJ, Gill JA (2013) Sex-biases in distribution and resource use at different spatial scales in a migratory shorebird. Ecol Evol 3:1079-1090

Birkhead TR, Nevo AJD (1987) Egg formation and the prelaying period of the common guillemot Uria aalge. J Zool (Lond) 211:83-88

Black JM (1996) Partnerships in birds: the study of monogamy. Oxford University Press, New York, NY

* Chastel O, Weimerskirch H, Jouventin P (1995) Influence of body condition on reproductive decision and reproductive success in the blue petrel. Auk 112:964-972

Choudhury S (1995) Divorce in birds: a review of the hypotheses. Anim Behav 50:413-429

Clutton-Brock TH (1989) Mammalian mating systems. Proc R Soc Lond B 236:339-372

* Conners MG, Hazen EL, Costa DP, Shaffer SA (2015) Shadowed by scale: subtle behavioral niche partitioning in two sympatric, tropical breeding albatross species. Mov Ecol 3:28

* Cornioley T, Börger L, Ozgul A, Weimerskirch H (2016) Impact of changing wind conditions on foraging and incubation success in male and female wandering albatrosses. J Anim Ecol 85:1318-1327

* Dean B, Freeman R, Kirk H, Leonard K, Phillips RA, Perrins CM, Guilford T (2012) Behavioural mapping of a pelagic seabird: combining multiple sensors and a hidden Markov model reveals the distribution of at-sea behaviour. J R Soc Interface 10:20120570

* Dubois F, Cézilly F (2002) Breeding success and mate retention in birds: a meta-analysis. Behav Ecol Sociobiol 52:357-364

Elliott KH, Ricklefs RE, Gaston AJ, Hatch SA, Speakman JR, Davoren GK (2013) High flight costs, but low dive costs, in auks support the biomechanical hypothesis for flightlessness in penguins. Proc Natl Acad Sci USA 110: 9380-9384

Emlen ST, Oring LW (1977) Ecology, sexual selection, and the evolution of mating systems. Science 197:215-223
Ens BJ, Choudhury S, Black JM (1996) Mate fidelity and divorce in monogamous birds. In: Black JM (ed) Partnerships in birds: the study of monogamy. Oxford University Press, Oxford, p 344-401

Fayet AL, Freeman R, Shoji A, Boyle D and others (2016) Drivers and fitness consequences of dispersive migration in a pelagic seabird. Behav Ecol 27:1061-1072

González-Solís J, Becker PH, Wendeln H (1999) Divorce and asynchronous arrival in common terns, Sterna hirundo. Anim Behav 58:1123-1129

Guilford T, Freeman R, Boyle D, Dean B, Kirk H, Phillips RA, Perrins CM (2011) A dispersive migration in the Atlantic puffin and its implications for migratory navigation. PLOS ONE 6:e21336

KGunnarsson TG, Gill JA, Sigurbjornsson T, Sutherland WJ (2004) Pair bonds: arrival synchrony in migratory birds. Nature 431:646

Gunnarsson TG, Gill JA, Atkinson PW, Gélinaud G and others (2006) Population-scale drivers of individual arrival times in migratory birds. J Anim Ecol 75:1119-1127

*Handel CM, Gill RE Jr (2000) Mate fidelity and breeding site tenacity in a monogamous sandpiper, the black turnstone. Anim Behav 60:471-481

Harris MP (1980) Breeding performance of puffins Fratercula arctica in relation to nest density, laying date and year. Ibis 122:193-209

Harris MP, Wanless S (2011) The puffin. T \& AD Poyser, London

Harris MP, Wanless S, Jensen JK (2014) When are Atlantic puffins Fratercula arctica in the North Sea and around the Faroe Islands flightless? Bird Study 61:182-192

* Jacobs SR, Elliott KH, Gaston AJ, Weber JM (2009) Fatty acid signatures of female Brünnich's guillemots Uria lomvia suggests reliance on local prey for replacement egg production. J Avian Biol 40:327-336

KLewis S, Benvenuti S, Dall'Antonia L, Griffiths R and others (2002) Sex-specific foraging behaviour in a monomorphic seabird. Proc R Soc Lond B 269:1687-1693

KLewis S, Phillips RA, Burthe SJ, Wanless S, Daunt F (2015) Contrasting responses of male and female foraging effort to year-round wind conditions. J Anim Ecol 84:1490-1496

* Müller MS, Massa B, Phillips RA, Dell'Omo G (2015) Seabirds mated for life migrate separately to the same places: behavioural coordination or shared proximate causes? Anim Behav 102:267-276

Naves LC, Cam E, Monnat JY (2007) Pair duration, breeding success and divorce in a long-lived seabird: benefits of mate familiarity? Anim Behav 73:433-444

Nettleship DN (1972) Breeding success of the common puffin (Fratercula arctica L.) on different habitats at Great Island, Newfoundland. Ecol Monogr 42:239-268

Newton I (2008) Migration ecology of birds. Academic Press, London

* Phillips RA, Silk JRD, Croxall JP, Afanasyev V, Briggs DR (2004) Accuracy of geolocation estimates for flying seabirds. Mar Ecol Prog Ser 266:265-272

* Phillips RA, McGill RAR, Dawson DA, Bearhop S (2011) Sexual segregation in distribution, diet and trophic level of seabirds: insights from stable isotope analysis. Mar Biol 158:2199-2208

Rowley I (1983) Re-mating in birds. In: Bateson P (ed) Mate choice. Cambridge University Press, New York, NY, p 331-360

Sánchez-Macouzet O, Rodriguez C, Drummond H (2014) Better stay together: pair bond duration increases indi- 
vidual fitness independent of age-related variation. Proc R Soc Lond B 281:20132843

Shaffer SA (2011) A review of seabird energetics using the doubly labeled water method. Comp Biochem Physiol A 158:315-322

Shoji A, Elliott K, Fayet A, Boyle D, Perrins C, Guilford T (2015) Foraging behaviour of sympatric razorbills and puffins. Mar Ecol Prog Ser 520:257-267

Shoji A, Dean B, Kirk H, Freeman R, Perrins CM, Guilford T (2016) The diving behaviour of the Manx shearwater Puffinus puffinus. Ibis 158:598-606

Editorial responsibility: Rory Wilson,

Swansea, UK
Thiebot JB, Bost CA, Dehnhard N, Demongin L and others (2015) Mates but not sexes differ in migratory niche in a monogamous penguin species. Biol Lett 11:20150429

WWendeln H, Becker PH (1999) Effects of parental quality and effort on the reproduction of common terns. J Anim Ecol 68:205-214

* Widmann M, Kato A, Raymond B, Angelier F and others (2015) Habitat use and sex-specific foraging behaviour of Adélie penguins throughout the breeding season in Adélie Land, East Antarctica. Mov Ecol 3:30

Submitted: August 9, 2016; Accepted: February 7, 2017

Proofs received from author(s): March 14, 2017 